\title{
Construction of a recombinant bacmid DNA containing influenza A virus hemagglutinin gene using a site-specific transposition mechanism
}

\author{
Shafaati $\mathrm{MR}^{1^{*}}$, Akhavan $\mathrm{E}^{2}$, Yazdani $\mathrm{SH}^{3}$, Shafaati $\mathrm{M}^{4}$ \\ ${ }^{1}$ Department of Cellular \& Molecular Biology IAU, Hamadan Branch, Hamadan, Iran. \\ ${ }^{2}$ Department of Microbiology IAU, Damaghan Branch, Damaghan, Semnan, Iran. \\ ${ }^{3}$ Department of Virology, School of Public Health, Tehran University of Medical Sciences, Tehran, Iran. \\ ${ }^{4}$ Department of Microbiology IAU, Jahrom Branch, Jahrom, Shiraz, Iran.
}

\begin{abstract}
Introduction: In recent years, influenza viruses have caused moderate to severe infections all around the world while so far there is no influenza vaccine that can protect people with only one dose of injection. In this regard, producing a universal vaccine based on virus-like-particles (VLP) could be an ideal approach. Methods: In this study, the fulllength ORF of influenza hemagglutinin (HA) gene from Influenza A virus of H9N2 subtype was amplified by RTPCR using specific primers to produce HA cDNA. The amplicon was cloned firstly into a T/A cloning vector and then was subcloned into a pFastBacDual donor plasmid through SalI/HindIII restriction sites. The recombinant HApFastBacDual vector was transferred to Escherichia coli DH10Bac cells, to insert the HA gene into the bacmid DNA via a site-specific transposition process. The recombinant bacmid was then extracted and further analyzed by PCR. Results: Our data indicated that the HA-containing recombinant bacmid was constructed successfully using the transposition mechanism between pFastBacDual-HA and the bacmid. Conclusion: The recombinant baculovirus construct in this work had proper characteristics to be used in production of H9N2 VLP in Sf9 insect cell line in the future studies.
\end{abstract}

KEYWORDS: Influenza A virus, Hemagglutinin protein, Baculovirus.

\section{INTRODUCTION}

Influenza A viruses are members of Ortomyxoviridae family and are able to infect a large variety of animals as well as humans. These viruses have a segmented-negative strand genome and are classified based on the antigenic properties of their two distinct surface glycoproteins. These influenza glycoproteins, namely, hemagglutinin (HA) and neuraminidase (NA) are located on the outer host-cell-derived envelope. Influenza A viruses of H9N2 subtype have become highly prevalent in poultry facilities in many countries. Although these viruses can generally cause only mild to moderate diseases, they can infect a wide variety of species, including chickens, quails, turkeys, ducks, geese, pheasants, partridges and pigeons [1-4]. More importantly, the occasional transmissions of H9N2 viruses from land-based poultry to humans and pigs have been reported [5-7]. Some investigations suggest that a significant proportion of H9N2 field isolates have acquired human viruslike receptors specificity. For instance, a few isolates that could

*Corresponding Author: Mohammad Reza Shafaati, Ph.D; Department of Cellular \& Molecular Biology IAU, Hamadan Branch, Hamadan, Iran.

Email: shafaati@iauh.ac.ir

Tel/Fax: (+98) 09362420374 recognize $\alpha$ 2, 6-linked sialic acid (SA $\alpha 2-6)$ have been transmitted directly to humans $[8-10,6,11]$. In addition to possessing human virus-like receptor specificities, avian H9N2 viruses induce a typical flu-like illness in humans which can easily go unnoticed. Eventually, Such viruses have the opportunity to circulate, resort and improve their transmissibility [10, 6, 12-14]. Based on these global concerns, scientific efforts have focused on the prevention and treatment of H9N2 avian influenza virus infections. The prevention method against the avian influenza is mainly through vaccination. Currently, most avian influenza vaccines used in the clinics are the inactivated types that have been propagated in embryonated chicken eggs. However, the use of inactivated avian influenza vaccines can induce little or no cellular immune responses and hence cannot provide a wide and persistent protection against influenza while it would interfere with the serological monitoring of the virus. In addition, egg-based influenza vaccine production is dependent on the availability of embryonated eggs which might be in limited supply in the events of severe influenza outbreaks or pandemics. In view of these potential drawbacks of the old vaccines, the development 
of a new type of H9N2 vaccine with emphasis on effectiveness and minimal side-effects and costs, is required.

From three decades ago, it has been shown that antibodies against the influenza hemagglutinin and neuraminidase can establish protection, similar to immunization induced with inactivated influenza vaccines $[1,2]$. One research avenue on this topic is the production of virus-like particles (VLPs). So far, VLPs have been expressed in by different methods and hosts which one of the best expression systems among them is the production of VLPs by baculoviruses in insect cells [15]. The combination of recombinant baculovirus and insect cell expression systems provides high levels of recombinant proteins that undergo post-translational modifications such as glycosylation [16, 17]. Therefore, the application of such systems allows to produce large quantities of the target protein in its native conformation as a vaccine [18].

In this study, HA gene was isolated and amplified from H9N2 influenza virus and then the HA-containing recombinant bacmid was constructed via a site-specific transposition. It is envisaged that such constructs can be used to produce recombinant protein in $S f 9$ insect cells [19]. Such HAcontaining recombinant bacmid could eventually be used to produce large scale preparation of hemagglutinin protein, suitable for influenza vaccine production.

\section{MATERIALS and METHODS}

Viral and bacterial strains and the plasmids

The virus strain in this study was A/Chicken/Iran/11T/99 (H9N2). Escherichia coli strain DH5a (Invitrogen, USA) was used for transformation and amplification of the recombinant vectors and E. coli strain DH10Bac (Invitrogen, USA) was used as an appropriate strain to perform the cloning processes. For cloning the HA gene, "T/A cloning vector" (RBC Bioscience, Taiwan) was used as the general vector. To generate the recombinant Bacmid, pFastBacDual was used as the transfer vector (Invitrogen, USA). E. coli strain DH10Bac contained the baculovirus modified DNA (Bacmid) with a mini-attTn7 target site and the helper plasmid. The helper plasmid harbored by DH10Bac strains, confers resistance to tetracycline and encodes enzymes needed for transposition of the gene of interest into the Bacmid.

\section{Design and synthesis of the primers}

All the sequences of HA protein in Iran were obtained from NCBI and were compared with each other. The amino acid sequences were converted into codons which were compatible for Sf9 cells using JAVA codon adaptation tool <http://www.genscript.com/cgi-bin/tools/rare_codon_analysis $>$. Cleavage sites for restriction enzymes SalI and HindIII were inserted at the beginning and the end of HA gene ORF and specific primers were designed by CLC Main Workbench 4.5 (QIAGEN Co.). The designed primers were synthesized by Metabion Company (Germany).

\section{In silico folding prediction of the recombinant HA protein}

The three-dimensional (3D) predictions of the recombinant HA protein folding were performed by Swissmodel, Raptorx and Antheprot 3D viewer web servers. Furthermore, the Ramachandran plots were depicted for each model by Rampage. The Ramachandran plot displays the main chain conformation angles (Phi and Psi) of the polypeptide chain of a protein molecule.

RNA extraction and cDNA synthesis
Viral RNA was extracted from $200 \mu$ of harvested cell culture supernatant using a Roche commercial kit (Germany) according to the manufacturer's instructions. The cDNA synthesis was performed using Thermo Script ${ }^{\mathrm{TM}}$ RT-PCR System (InvitrogenUSA) according to the manufacturer's instructions using specific primers (at the concentration of $10 \mu \mathrm{M}$ or $10 \mathrm{pmol} / \mu \mathrm{l}$ for each primer), dNTPs (10 mM for each base) and RNAase inhibitor in RNAase/DNase-free microtubes (Extra gene, USA).

\section{RT-PCR}

Specific primers (forward and reverse) were designed by CLC Main Workbench 4.5 (QIAGEN Co.) in order to amplify the complete HA gene ORF of H9N2 influenza (1682 bp). Five $\mu \mathrm{l}$ of cDNA were added to a $20 \mu$ l total volume of PCR mixture containing 10 pmol of each forward primer 5' GTC-GAC-ATGGAA-ACA-ATA-TCA-CT 3' and reverse primer 5' AAG-CTTTTA-TAT-ACA-AAT-GTT-GCA-CC 3' nucleotides correspond to SalI and HindIII restriction sites, respectively), $10 \mathrm{mM}$ Tris- $\mathrm{Cl} \mathrm{pH} 8.3,50 \mathrm{mM} \mathrm{KCl}, 1.5 \mathrm{mM}$ $\mathrm{MgCl} 2,10 \mathrm{mM}$ dNTPs and high fidelity PCR Enzyme Mix (Genet Bio, Korea). The amplification reactions were performed in a thermocycler (ABI, USA) using the following program: $5 \mathrm{~min}$ at $95^{\circ} \mathrm{C}$ followed by 30 cycles at $95^{\circ} \mathrm{C}$ for 30 sec, $50^{\circ} \mathrm{C}$ for $30 \mathrm{sec}$ and $72^{\circ} \mathrm{C}$ for $80 \mathrm{sec}$, with a final extension step at $72^{\circ} \mathrm{C}$ for $10 \mathrm{~min}$. The PCR products were analyzed on $1 \%(\mathrm{w} / \mathrm{v})$ electrophoresis agarose gel stained with safe view (Kiagene, IRI) [20, 21].

\section{Cloning of HA gene}

The RT-PCR product was extracted from low melting agarose gel using a DNA extraction kit (Vivantis, Korea) and the amplicon was subsequently cloned into the T/A cloning vector and then subcloned into the pFastBacDual donor plasmid through SalI/HindIII restriction sites. The HA-containing recombinant pFastBacDual donor vector was confirmed using PCR and enzymatic digestion analysis. Subsequently, it was subjected to perform automatic one-directed sequencing, using the forward and the reverse primers as described above for the HA gene amplification.

\section{Generation of the recombinant bacmid}

The HA-containing recombinant pFastBacDual donor plasmid was transferred into E. coli $\mathrm{DH} 10 \mathrm{Bac}$ competent cells for sitespecific transposition of the HA DNA from the transposing vector to a Bacmid DNA through lacZ gene disruption. The transformed cells were cultured on a LB agar plate containing kanamycin $(50 \mu \mathrm{g} / \mathrm{ml})$, gentamicin $(7 \mu \mathrm{g} / \mathrm{ml})$, tetracycline $(10$ $\mu \mathrm{g} / \mathrm{ml})$, X-gal $(100 \mu \mathrm{g} / \mathrm{ml})$ and isopropylthio- $\beta$-galactoside (IPTG, $40 \mu \mathrm{g} / \mathrm{ml}$ ) and incubated at $37^{\circ} \mathrm{C}$ for $24 \mathrm{~h}$. The highmolecular-weight bacmid DNA was isolated from the overnight cultures by an alkaline lysis purification method, according to the instructions supplied by the manufacturer (InvitrogenUSA). Successful transposition was verified by PCR analysis using both M13/pUC and HA specific primers.

\section{RESULTS}

\section{Codon optimization and structural prediction of HA protein}

Codons of the selected sequence of HA protein from H9N2 influenza strains isolated in Iran were analysed for their expression in $S f 9$ insect cells by online software tools and expression of this gene in the insect cells was optimized by converting the codons into those suitable for expression by $S f 9$ cells which resulted in Codon Adaptation Index (COI) of 0.84 . (Fig. 1). The optimized sequence was re-investigated in terms 
of average GC content which was determined to be $42.38 \%$ as shown in Fig. 2. Therefore, the most appropriate GC-content and the highest value of adaptation with codons were used in order to express the gene in $S f 9$ cells. The study of the protein's secondary structure using SOPMA program predicted that the protein product of the gene of interest was containing 116 alpha helices (Hh), 147 extended strands (ES) and 191 random coils

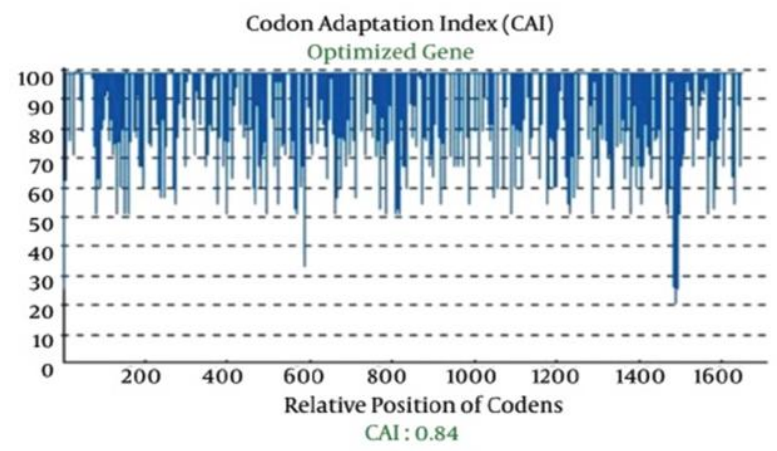

Fig. 1. The distribution of codon usage frequency along the length of the target gene using GenScript rare codon analysis tool. The possibility of high protein expression level is correlated to the value of CAI. A CAI of 1.0 is considered to be ideal while a CAI of $>0.8$ is rated as good for expression in the desired expression organism.
(CC) as depicted in Fig. 3A. The 3D structure of the protein, predicted by Antheprot 3D viewer program is shown in Fig. 3B. The Ramachandran plot (Fig. 3C) of the refined model estimated that the number of residues in the favored region to be $141.5(98.8 \%)$, the number of residues in the allowed region to be $2(1.2 \%)$ and the number of residues in the outlier region to be $0(0 \%)$.

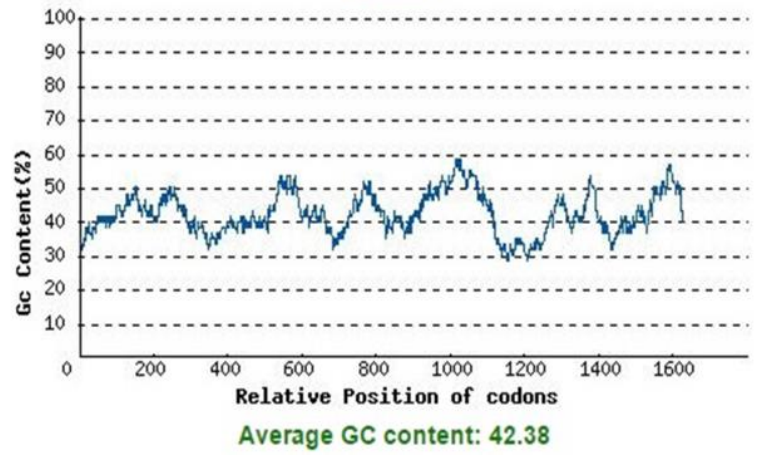

Fig. 2. The primary sequence of the designed gene using GenScript rare codon analysis tool which was adapted to usable codons in Sf9 cells. The ideal percentage range of GC content is between 30-70\%. Any peaks outside of this range will adversely affect the transcriptional and the translational efficiency.
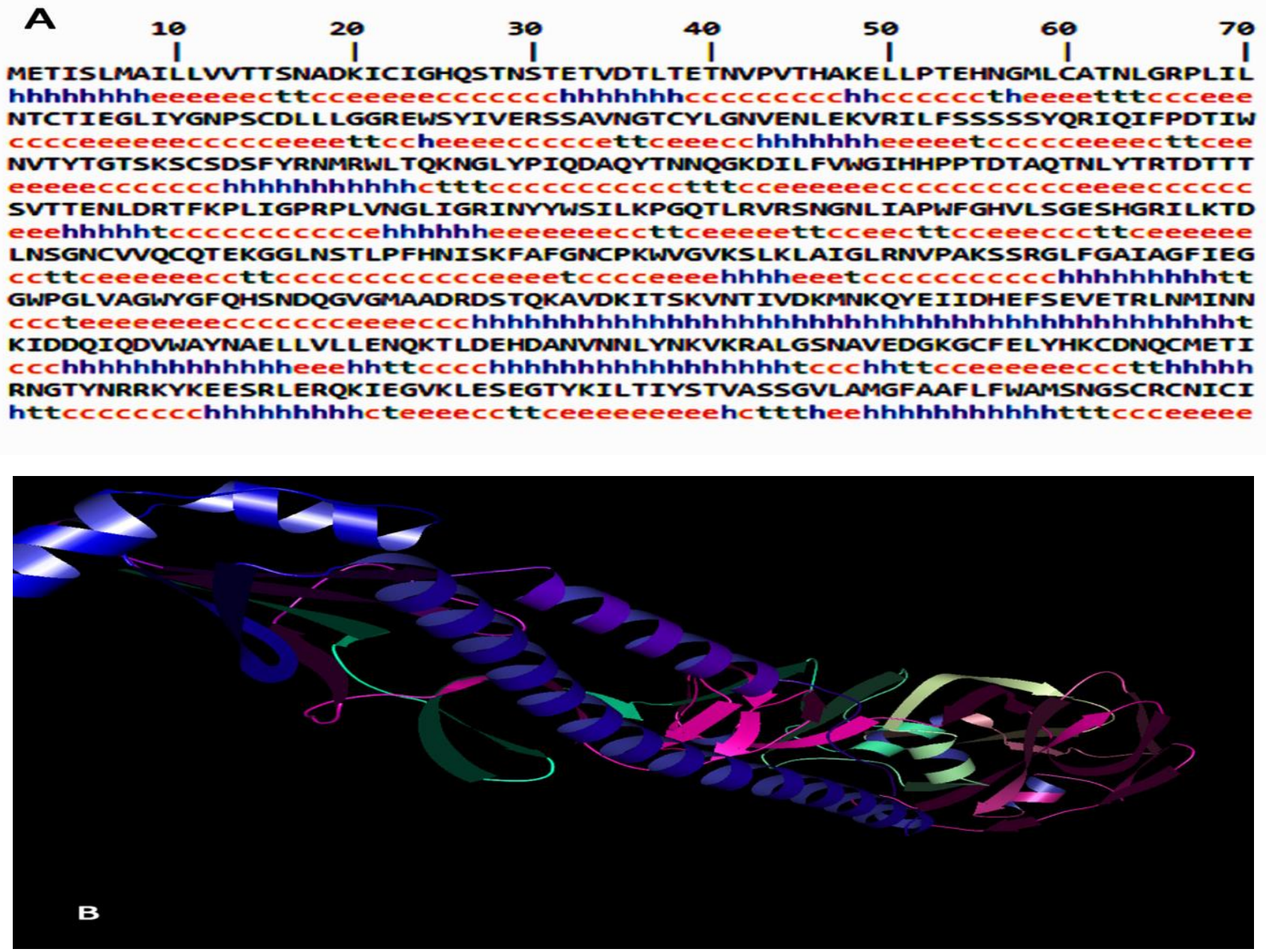


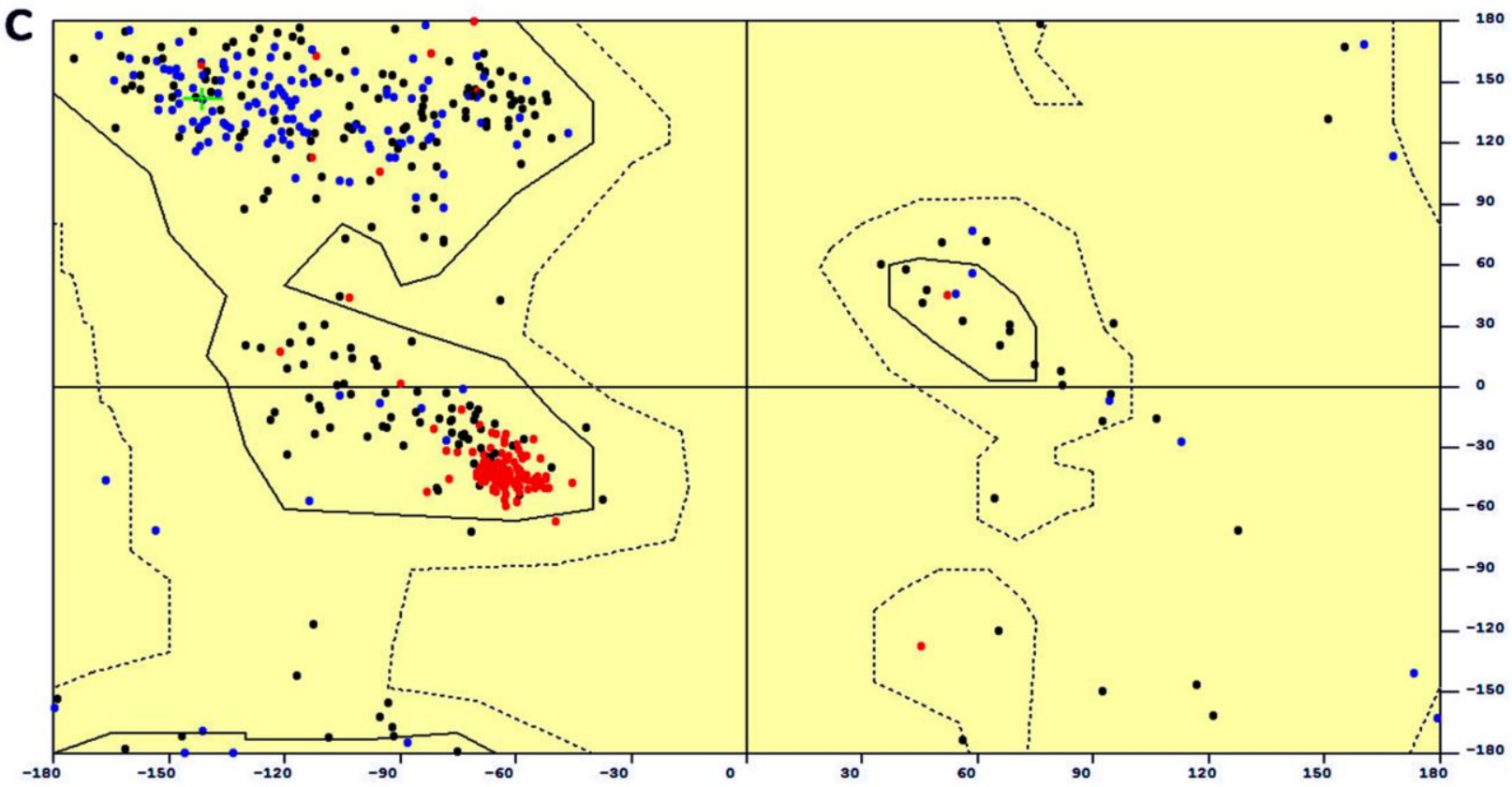

Fig. 3. A) The predicted secondary structure of HA protein: Alpha helices (Hh), extended strands (ES), random coils (CC). B) The predicted 3D structure model of HA protein. C) The Ramachandran plot of the refined model using Antheprot 3D viewer software. The plot predicts that the HA protein structure has $100 \%$ of its residues in most favored areas, resulting in Z-scores of -0.98 . The blue color is used for the helices, the red for the strands and the green for the turns and loops.

\section{RT-PCR and T/A cloning}

The full-length sequence of HA gene with an expected size of 1686 bp was amplified by RT-PCR using the designed specific primers (Fig. 4). The amplicon was cloned into a T/A cloning vector after extraction from the agarose gel and was confirmed by PCR (Fig. 5A) and enzymatic digestion analysis (Fig 5B). The ORF accuracy of the HA gene in T/A cloning vector was confirmed by nucleotide sequencing. The sequencing analysis was accomplished by Chromas software (version 1.45, Australia). Subsequently, the HA fragment was subcloned into the pFastBacDual donor plasmid, as described previously in the methods section. The integrity of the recombinant vector was confirmed by PCR and subsequently by enzymatic digestion (results not shown).

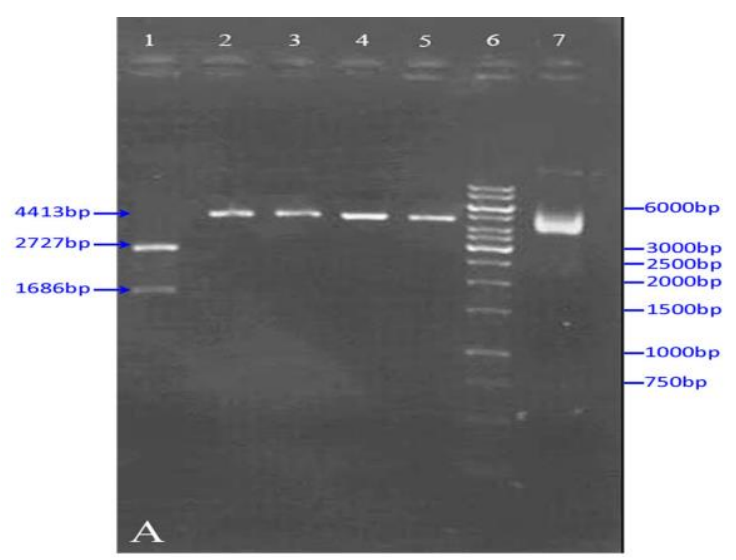

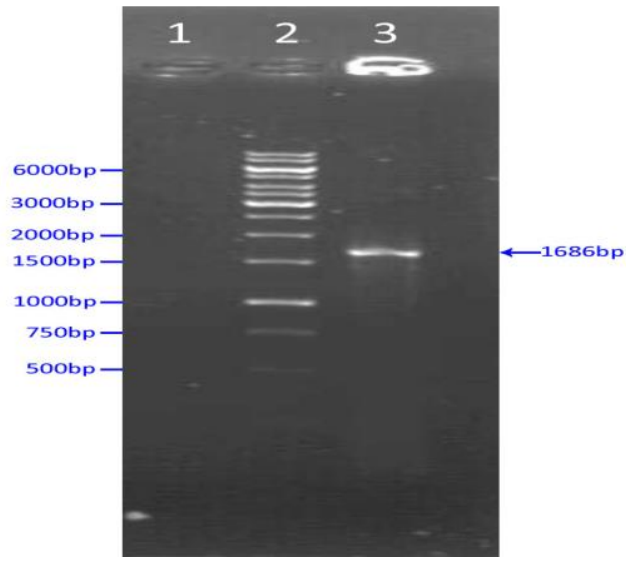

Fig. 4. Gel electrophoresis analysis of HA gene RT-PCR product: Lane 1: Negative control. Lane 2: $1 \mathrm{~Kb}$ DNA ladder (Fermentas \#SM0311). Lane 3: The amplicon (expected size: $1686 \mathrm{bp}$ ).

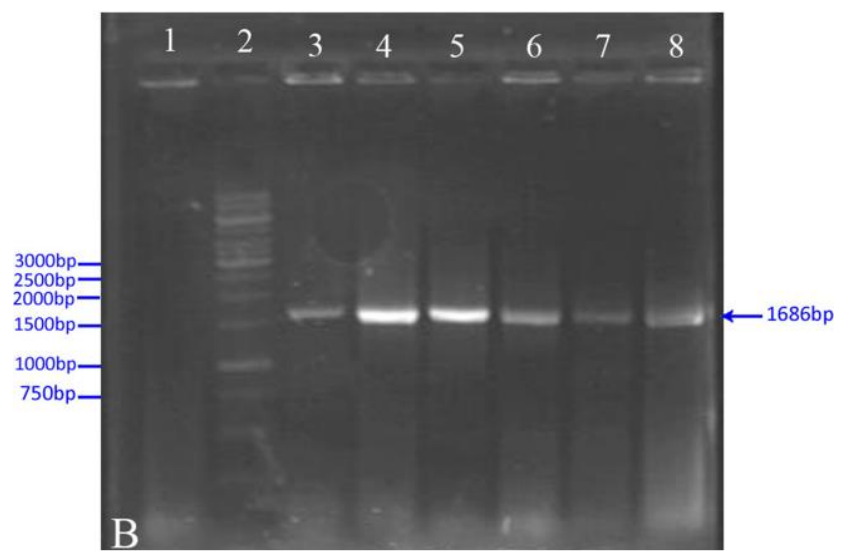

Fig.5. A) Enzymatic digestion map of the new HA-containing recombinant T/A cloning vector. Lane 6: $1 \mathrm{~Kb}$ DNA size marker (Fermentas \#SM0311). Lane 1: SalI/HindIII double digestion on the HA-containing recombinant T/A cloning vector (clone), revealed expected 2727 bp and 1686 bp fragments. Lanes 2-3: single digested with HindIII enzyme fragments (expected size: 4413 bp). Lanes 4-5: single digested with SalI enzyme fragments (expected size: $4413 \mathrm{bp}$ ). Lane 7: undigested plasmid of the new HA-containing recombinant T/A cloning vector. B) HA, gene PCR with specific primer, six white colonies were selected randomly. Lane 1: negative control (blank) Lane 2: 1 Kb DNA size marker (Fermentas \#SM0311). Lanes 3-8: HA-gene fragment (1686 bp). 


\section{Construction of the recombinant bacmid DNA}

The HA gene was subcloned into a pFastBacDual donor plasmid and the construct was verified by endonuclease digestion in comparison with a non-recombinant pFastBacDual vector, as a control. Following the transformation and plating of the cells on X-gal/IPTG LB agar, the transposed colonies were visible as large white colonies among the blue ones which harbored the unaltered bacmids. The selected white colonies were re-streaked on the agar plates to ensure whether they had a true white phenotype. Since verification of the high molecular weight recombinant bacmid DNA is not convenient by digestion, PCR was performed using both M13/pUC and HAspecific primers to ensure the proper transposition of the target gene in the recombinant bacmid. The bacmid DNA contains M13 forward and reverse priming sites, flanking the Tn7 miniatt site within the LacZ $\alpha$-complementation region. A panel of PCR was done using M13/pUC universal primers, HA gene specific forward and M13 reverse primers, HA gene specific reverse and M13 forward primers, and finally HA gene specific primers, respectively. The amplification of the non-recombinant bacmid using M13/pUC primers generated a band with estimated size of 303 bp (Fig. 6).

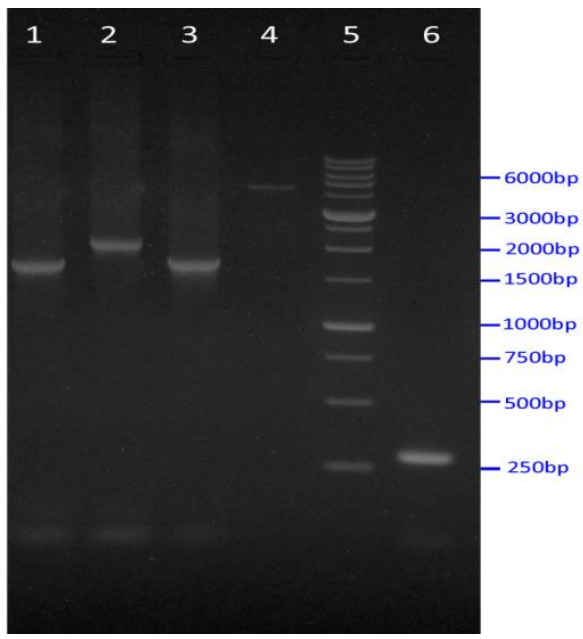

Fig. 6. PCR panel showing HA-containing recombinant Bacmid: Lane 5: The $1 \mathrm{~Kb}$ DNA ladder. Lane 1: PCR product using HA gene specific primers (expected size: $1686 \mathrm{bp}$ ) produced by the recombinant bacmid extracted from the same white colony. Lane 2: PCR product using M13 forward and HA reverse primers (expected size: $\sim 2200 \mathrm{bp}$ ) produced by the recombinant bacmid extracted from the same white colony. Lane 3: PCR product using HA gene forward and M13 reverse primers (expected size: $\sim 1500 \mathrm{bp}$ ) produced by the recombinant bacmid extracted from the same white colony. Lane 4: PCR product using M13 specific primers (expected size: $4246 \mathrm{bp}$ ) produced by the recombinant bacmid extracted from a white colony. Lane 6: PCR product using M13 universal primers (expected size: $\sim 300 \mathrm{bp}$ ) produced by the nonrecombinant bacmid extracted from a blue colony as a negative control.

\section{DISCUSSION}

The expression of genes using baculovirus expression vectors in eukaryotic insect cells takes advantages of the host's protein synthesis machinery and facilitates proper folding and posttranslational modifications, including glycosylation, acetylation, oligomerization and proteolysis. In addition, the cytoplasmic environment of the insect cell provides appropriate disulfide bridge assemblies [21, 22, 18, 23]. These features are crucial for expression of influenza HA-protein where its proper structure is critical for its function as a nexus that gathers ribonucleoprotein complex and outer envelope-bound NA and $\mathrm{M}$ proteins together for packaging in the host cell [24, 25]. The production of influenza VLPs have been demonstrated with different expression systems including recombinant DNA expression vectors [20], recombinant vaccinia viruses [25, 18], and DNA plasmid transfection with T7 RNA polymeraseexpressing vaccinia virus [24]. However, recombinant baculovirus/insect cell expression systems which yields high levels of recombinant proteins and is considered to be more important. VLPs formed in such systems have shown high immunogenicity both in the raise of neutralizing antibodies and the induction of cellular immune responses. Several research groups have reported the expression of influenza HA-gene in baculovirus-infected insect cells and most of them had planned to produce various Influenza VLPs of different genotypes for application in vaccine research studies [26, 27, 21, 28]. For instance, Gomez-Puerto and colleagues have clearly demonstrated the significance of HA protein in assembly and the process of influenza VLP formation [24, 29]. Therefore, in most influenza VLP studies, the HA protein has been considered as an essential component [20, 22, 27, 28]. It has also been demonstrated that VLPs lacking NA have been formed and released from the cells expressing HA through recombinant DNA or virus infection [25].

In this study, a recombinant baculovirus containing a correct and in-frame sequence of influenza HA gene under the control of polyhedrin promoter was constructed. To produce influenza VLPs as an ultimate goal, we chose the Bac-to-Bac expression system (Invitrogen, USA) because apart from the abovementioned advantages, it is a valid and multipurpose system for the production of recombinant proteins. In future studies, this recombinant baculovirus expressing hemagglutinin gene could also be used with other individual recombinant baculoviruses expressing NA and M genes to produce influenza VLPs. It is envisaged that the proteins resulted from these constructs could be purified for influenza vaccine research studies.

\section{ACKNOWLEDGEMENT}

The authors would like to thank the Applied Virology Research Center at Baqiyatallah University of Medical Sciences, for the moral support and services provided during this project.

\section{CONFLICT OF INTEREST}

The authors declare that they have no conflict of interest.

\section{REFERENCES}

1. Alexander DJ. A review of avian influenza in different bird species. Veterinary microbiology. 2000;74(1):3-13.

2. Guan Y, Shortridge KF, Krauss S, Webster RG. Molecular characterization of H9N2 influenza viruses: were they the donors of the "internal" genes of H5N1 viruses in Hong Kong. Proceedings of $t$ he National Academy of Sciences. 1999;96(16):9363-7.

3. Munster V, Fouchier R. Avian influenza virus: of virus and bird ecology. Vaccine. 2009;27(45):6340-4.

4. Tang X, Tian G, Zhao J, Zhou K, Yu K. Isolation and characterization of prevalent strains of avian influenza viruses in China. Chinese Journal of Animal and Poultry Infectious Diseases. 1998;20:1-5.

5. Guo Y, Li J, Cheng X. Discovery of men infected by avian influenza A (H9N2) virus. Chinese journal of experimental and clinical virology. 1999;13(2):105-8.

6. Peiris J, Guan Y, Markwell D, Ghose P, Webster R, Shortridge K. Cocirculation of avian H9N2 and contemporary "human" H3N2 influenza A 
viruses in pigs in southeastern China: potential for genetic reassortment. Journal of virology. 2001;75(20):9679-86.

7. Peiris M, Yuen K, Leung C, Chan K, Ip P, Lai R et al. Human infection with influenza H9N2. The Lancet. 1999;354(9182):916-7.

8. Butt K, Smith GJ, Chen H, Zhang L, Leung YC, Xu K et al. Human infection with an avian H9N2 influenza A virus in Hong Kong in 2003. Journal of clinical microbiology. 2005;43(11):5760-7.

9. Choi Y, Ozaki H, Webby R, Webster R, Peiris J, Poon L et al. Continuing evolution of H9N2 influenza viruses in Southeastern China. Journal of virology. 2004;78(16):8609-14.

10. Li K, Xu K, Peiris J, Poon L, Yu K, Yuen K et al. Characterization of H9 subtype influenza viruses from the ducks of southern China: a candidate for the next influenza pandemic in humans. Journal of virology. 2003;77(12):6988-94

11. Saito T, Lim W, Suzuki T, Suzuki Y, Kida H, Nishimura S-I et al. Characterization of a human H9N2 influenza virus isolated in Hong Kong. Vaccine. 2001;20(1):125-33.

12. Sun Y, Pu J, Jiang Z, Guan T, Xia Y, Xu Q et al. Genotypic evolution and antigenic drift of H9N2 influenza viruses in China from 1994 to 2008. Veterinary microbiology. 2010;146(3):215-25.

13. Xu K, Li K, Smith G, Li J, Tai H, Zhang J et al. Evolution and molecular epidemiology of H9N2 influenza A viruses from quail in southern China, 2000 to 2005. Journal of virology. 2007;81(6):2635-45.

14. Zhang P, Tang Y, Liu X, Liu W, Zhang X, Liu H et al. A novel genotype H9N2 influenza virus possessing human H5N1 internal genomes has been circulating in poultry in eastern China since 1998. Journal of virology. 2009;83(17):8428-38.

15. Guillen G, Aguilar J, Duenas S, Hermida L, Guzmán M, Penton E et al. Virus-like particles as vaccine antigens and adjuvants: application to chronic disease, cancer immunotherapy and infectious disease preventive strategies. Procedia in Vaccinology. 2010;2(2):128-33.

16. Anderson D, Harris R, Polayes D, Ciccarone V, Donahue R, Gerard G et al. Rapid generation of recombinant baculovirus and expression of foreign genes using the Bac-to-Bac baculovirus expression system. Focus. 1995;17(2):53-8.

17. Luckow VA, Lee S, Barry G, Olins P. Efficient generation of infectious recombinant baculoviruses by site-specific transposon-mediated insertion of foreign genes into a baculovirus genome propagated in Escherichia coli. Journal of virology. 1993;67(8):4566-79.

18. Shafaati Mr, Moghbeli M, Dorostkar R. Construction of Recombinant
Bacmid DNA Encoding Newcastle Disease Virus (NDV) Fusion Protein Gene. Iranian Journal of Virology 2013;7(1\&2):6.

19. Tan P, Khan AM, August JT. Highly conserved influenza A sequences as $\mathrm{T}$ cell epitopes-based vaccine targets to address the viral variability. Hum Vaccin. 2011;7(4):402-9.

20. Chen BJ, Leser GP, Morita E, Lamb RA. Influenza virus hemagglutinin and neuraminidase, but not the matrix protein, are required for assembly and budding of plasmid-derived virus-like particles. Journal of virology. 2007;81(13):7111-23.

21. Quan F-S, Huang C, Compans RW, Kang S-M. Virus-like particle vaccine induces protective immunity against homologous and heterologous strains of influenza virus. Journal of virology. 2007;81(7):3514-24.

22. Li B, Wu H-Y, Qian X-P, Li Y, Chen W-F. Expression, purification and serological analysis of hepatocellular carcinoma associated antigen HCA587 in insect cells. WORLD JOURNAL OF GASTROENTEROLOGY. 2003;9(4):678-82.

23. Najafi S, Behzadian F, Fotuhi F, Fallah Mehrabadi J. Construction of a recombinant bacmid DNA in order to express Neuraminidase gene of influenza virus H1N1. Arak University of Medical Sciences Journal. 2012;15(5):58-65.

24. Gómez-Puertas P, Albo C, Pérez-Pastrana E, Vivo A, Portela An. Influenza virus matrix protein is the major driving force in virus budding. Journal of virology. 2000;74(24):11538-47.

25. Ali A, Avalos RT, Ponimaskin E, Nayak DP. Influenza virus assembly: effect of influenza virus glycoproteins on the membrane association of M1 protein. Journal of virology. 2000;74(18):8709-19.

26. Kang S-M, Yoo D-G, Lipatov AS, Song J-M, Davis CT, Quan F-S et al. Induction of long-term protective immune responses by influenza $\mathrm{H} 5 \mathrm{~N} 1$ virus-like particles. PLoS One. 2009;4(3):e4667.

27. Mahmood K, Bright RA, Mytle N, Carter DM, Crevar CJ, Achenbach JE et al. H5N1 VLP vaccine induced protection in ferrets against lethal challenge with highly pathogenic H5N1 influenza viruses. Vaccine. 2008;26(42):5393-9.

28. Wang B-Z, Quan F-S, Kang S-M, Bozja J, Skountzou I, Compans RW. Incorporation of membrane-anchored flagellin into influenza virus-like particles enhances the breadth of immune responses. Journal of Virology. 2008;82(23):11813-23.

29. Mena I, Castillo M, Vivo A, Portela A. Efficient formation of influenza virus-like particles: dependence on the expression levels of viral proteins. Journal of General Virology. 1999;80(7):1635-45. 